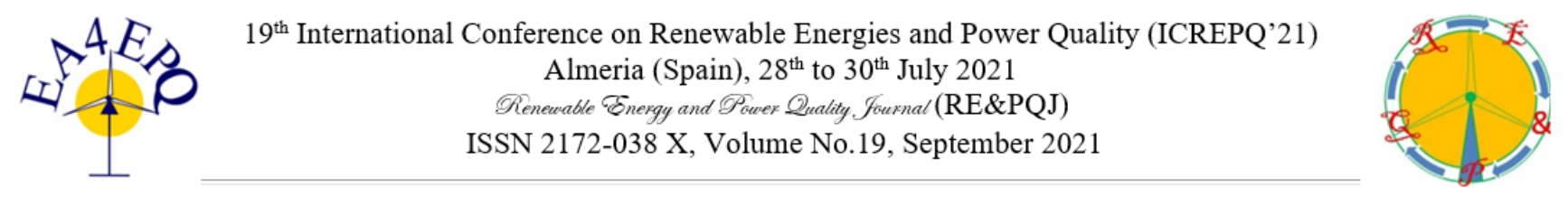

\title{
Three-phase Quaternion Power in Three-wire Systems
}

\author{
André S. F. Komeno, Anésio L. F. Filho, João Y. Ishihara and Victor P. Brasil \\ Department of Electrical Engineering \\ University of Brasilia \\ Campus Darcy Ribeiro - Brasilia (Brazil) \\ Phone number: +55 61984230003 \\ e-mail: aseikifk@gmail.com, leles@ene.unb.br, ishihara@ene.unb.br, vpradobrasil@gmail.com
}

\begin{abstract}
Instantaneous power theory has a central role in power systems analysis. Among mathematical settings used for the development of this theory, quaternion algebra has been used for describing electrical variables in recent works. In this context, this paper aims to describe three-phase power in a quaternion framework. We analyze quaternion power for balanced and unbalanced delta loads, comparing the expressions obtained to the usual expressions of complex power. The quaternion power expression obtained also makes it natural to introduce a decomposition of the unbalanced load in terms of a balanced component and an unbalanced load with null average power. It is also shown that delta unbalanced loads are equivalent to time-varying balanced loads. The results obtained extend the power systems theory in the quaternion domain and emphasize the advantages of using this framework.
\end{abstract}

Keywords. Instantaneous Active and Reactive Power, Quaternion Electrical Quantities, Three-Phase Three-Wire Circuits, Loads equivalents, Power systems theory

\section{Introduction}

For analysis of power systems under unbalanced conditions, there are several approaches for the characterization of electrical voltage, current, and power [1]-[11]. These characterizations can be classified into two different domains: the time-domain and the frequency domain.

Particularly, the time-domain characterization has been utilized to develop powerful analysis tools [1]-[11]. In [1], the instantaneous p-q power theory is proposed employing the Clark transformation of instantaneous voltages and currents of a three-phase system. This theory allows the compensation of reactive power without energy storage components. The theory was extended by [2]. The author presented another interpretation that allows instantaneous reactive power characterization without the need of performing a Clark transformation. It makes possible analysis of polyphase networks. In [3], a generalized theory of instantaneous reactive power is proposed and analyzed for its physical meaning.

All the above mentioned authors considered instantaneous space vectors to represent voltages and currents. More recently, some researchers have investigated instantaneous power quantities using a more general mathematical setting such as geometric algebra, tensor analysis, and quaternion algebra [4]-[11].

A framework based on quaternions seems to be promising for analysis of several problems in power systems and has already produced some interesting results [8]-[11]. In [8], the electromagnetic transients in a three-phase squirrel-cage induction motor were analyzed. The authors of [9] analyze a single-phase RLC circuit. In [10], it is pointed out that quaternion characterization of three-phase quantities serves as a link between the usual time and frequency domains. More specifically, with quaternions, it is possible to define three-phase electrical quantities analogously to singlephase's with phasors. In [11], quaternions were applied for instantaneous compensation of distorting effects in an inverter. 
In this paper, we extend the previous work of [10] for quaternion power analysis, highlighting the relation between power and load. In [10], the quaternionic power for a load was described in terms of quaternionic impedance but only for a balanced load in Y configuration. Here, we study the quaternionic power for a general (balanced and unbalanced) load in $\Delta$ configuration.

The rest of this paper is organized as follow. Section 2 presents quaternions definition and properties. In section 3 the expression of quaternionic power for three-phase balanced and unbalanced cases in three wire systems is determined. These expressions are compared to complex power expressions. Finally, section 4 presents conclusions and suggestions for future works.

\section{Quaternion Definition and Properties}

Originally proposed by Hamilton who was trying to write a rotation of an element in $\mathbb{R}^{3}$, quaternions are a mathematical group denoted by $\mathbb{H}$. They are referred to as hypercomplex numbers and can be defined as

$$
A=r+x \boldsymbol{q}_{1}+y \boldsymbol{q}_{2}+z \boldsymbol{q}_{3}
$$

where $r, x, y$ and $z$ are real numbers and $q_{1}, q_{2}$ and $q_{3}$ form, along with 1 , the orthonormal basis of the hypercomplex space. This basic quaternion are related according to the famous expression discovered by Hamilton

$$
\boldsymbol{q}_{1}^{2}=\boldsymbol{q}_{2}^{2}=\boldsymbol{q}_{3}^{2}=\boldsymbol{q}_{1} \boldsymbol{q}_{2} \boldsymbol{q}_{3}=-1
$$

\section{A. Scalar and vectorial parts}

Analogously to complex numbers real and imaginary parts, quaternions can be separated into scalar and vectorial parts. Considering the quaternion presented in (1), its scalar part $\tilde{\boldsymbol{A}}$ and vectorial part $\vec{A}$ are, respectively, given by

$$
\begin{gathered}
\tilde{\boldsymbol{A}}=r \\
\overrightarrow{\boldsymbol{A}}=x \boldsymbol{q}_{1}+y \boldsymbol{q}_{2}+z \boldsymbol{q}_{3} .
\end{gathered}
$$

\section{B. Product}

In quaternion algebra, product of two quaternions $\boldsymbol{A}$ and $\boldsymbol{B}$ is non-commutative and defined by

$$
\boldsymbol{A} \boldsymbol{B}=(\widetilde{\boldsymbol{A}} \widetilde{\boldsymbol{B}}-\overrightarrow{\boldsymbol{A}} \cdot \overrightarrow{\boldsymbol{B}})+(\widetilde{\boldsymbol{A}} \overrightarrow{\boldsymbol{A}}+\widetilde{\boldsymbol{B}} \overrightarrow{\boldsymbol{B}}+\overrightarrow{\boldsymbol{A}} \times \overrightarrow{\boldsymbol{B}})
$$

where "." and " $\times$ " represents, respectively, the dot product and the cross product. In particular, if $\boldsymbol{A}$ and $\boldsymbol{B}$ are pure vectorial quaternions (scalar part equal to zero), then their product can be simplified to

$$
A B=-\vec{A} \cdot \vec{B}+\vec{A} \times \vec{B} .
$$

\section{Conjugate}

Analogously to complex numbers, quaternions also have a conjugate. The conjugate of the quaternion $\boldsymbol{A}$ is the quaternion

$$
\boldsymbol{A}^{*}=\widetilde{\boldsymbol{A}}-\overrightarrow{\boldsymbol{A}} \text {. }
$$

\section{Norm}

The norm of a quaternion $\boldsymbol{A}$ is its Euclidean distance to the origin. It is defined as

$$
|\boldsymbol{A}|=\sqrt{r^{2}+x^{2}+y^{2}+z^{2}} .
$$

\section{E. Inverse}

The inverse of a quaternion $\boldsymbol{A}$ is defined in terms of its conjugate and norm according to

$$
\boldsymbol{A}^{-1}=\frac{\boldsymbol{A}^{*}}{|\boldsymbol{A}|^{2}} .
$$

\section{F. Polar form}

The polar form of a quaternion $\boldsymbol{A}$ similarly to complex numbers is defined in terms of its norm and an angle $\theta$. If $\vec{d}$ is the purely vectorial unitary quaternion in the same direction of $\vec{A}$ and $\theta$ the angle between $\vec{d}$ and the real axis, the polar form is defined as

$$
\boldsymbol{A}=|\boldsymbol{A}| e^{\overrightarrow{\boldsymbol{d}} \theta}=|\boldsymbol{A}|(\cos (\theta)+\overrightarrow{\boldsymbol{d}} \sin (\theta)) .
$$

\section{Quaternion power in three-wire systems}

For defining the quaternionic power in an $\mathrm{ABC}$ three-phase sinusoidal system, first we define quaternion voltage and current. The quaternion balanced voltage can be defined according to [10] by

$$
\boldsymbol{V}(t)=v_{a}(t) \boldsymbol{q}_{1}+v_{b}(t) \boldsymbol{q}_{2}+v_{c}(t) \boldsymbol{q}_{3}
$$

where

$$
\begin{gathered}
v_{a}(t)=V_{o} \cos (\omega t) \\
v_{b}(t)=v_{a}(t-2 \pi / 3 \omega) \\
v_{c}(t)=v_{a}(t+2 \pi / 3 \omega)
\end{gathered}
$$

represents instantaneous voltages of each phase, $V_{o}$ is the voltage amplitude in $\mathrm{V}, t$ is the time in $\mathrm{s}$, and $\omega$ is the electrical frequency in $\mathrm{rad} / \mathrm{s}$.

Analogously, the three-phase current quaternion are defined by

$$
\boldsymbol{I}(t)=i_{a}(t) \boldsymbol{q}_{1}+i_{b}(t) \boldsymbol{q}_{2}+i_{c}(t) \boldsymbol{q}_{3}
$$

in which $i_{a}(t), i_{b}(t)$, and $i_{c}(t)$ are line currents flowing through phases $\mathrm{A}, \mathrm{B}$ and $\mathrm{C}$, respectively.

The power quaternion is defined in [10] as

$$
\boldsymbol{S}(t)=\boldsymbol{V}(t) \boldsymbol{I}^{*}(t) .
$$

It can also be written as

$$
\boldsymbol{S}(t)=p_{a b c}(t)-\overrightarrow{\boldsymbol{Q}}(t)
$$

where $p_{a b c}(t)$ is the instantaneous three-phase active power and instantaneous reactive power is

$$
\overrightarrow{\boldsymbol{Q}}(t)=q_{a}(t) \boldsymbol{q}_{1}+q_{b}(t) \boldsymbol{q}_{2}+q_{c}(t) \boldsymbol{q}_{3}
$$




$$
\begin{aligned}
& q_{a}(t)=v_{b}(t) i_{c}(t)-v_{c}(t) i_{b}(t) \\
& q_{b}(t)=v_{c}(t) i_{a}(t)-v_{a}(t) i_{c}(t) \\
& q_{c}(t)=v_{a}(t) i_{b}(t)-v_{b}(t) i_{a}(t) .
\end{aligned}
$$

\section{A. Three-phase balanced loads}

Using these definitions, in [10] a three-phase series RLC balanced circuit is analysed and the power quaternion for the Y load is obtained.

$$
\boldsymbol{S}(t)=|\boldsymbol{V}(t)|^{2} \frac{\boldsymbol{Z}}{|\boldsymbol{Z}|^{2}}
$$

where

$$
\boldsymbol{Z}=R+\overrightarrow{\boldsymbol{n}}\left(\omega L-\frac{1}{\omega C}\right)
$$

in which $\vec{n}=1 / \sqrt{3}\left(\boldsymbol{q}_{1}+\boldsymbol{q}_{2}+\boldsymbol{q}_{3}\right)$ is a unitary quaternion.

The quaternion impedance in (23) can be rewritten in its polar for as

$$
\boldsymbol{Z}=|\boldsymbol{Z}|(\cos (\theta)+\overrightarrow{\boldsymbol{n}} \sin (\theta))=|\boldsymbol{Z}| e^{\overrightarrow{\boldsymbol{n}} \theta}
$$

where $\cos (\theta)$ is the power factor of the series RLC impedance. Notice that the angle $\theta$ in (24) is equal to the phase of the phasor representation of RLC impedance and $|\boldsymbol{Z}|$ is equal to its absolute value.

From now on, we extend the power analysis of [10] and analyze it for a general balanced or unbalanced $\Delta$ loads represented by admittances.

First, considering the same load in [10] and using (24) in (22) we obtain

$$
\boldsymbol{S}(t)=Y|\boldsymbol{V}(t)|^{2} e^{\overrightarrow{\boldsymbol{n}} \theta}
$$

where $Y=|\boldsymbol{Z}|^{-1}$ is the absolute value of the load quaternion admittance.

Let us consider the $\mathrm{Y}-\Delta$ transform of this load. In this case, $Y=3 Y_{\Delta}$. Considering (11), quaternion power can be expressed by

$$
\boldsymbol{S}=\frac{9}{2} V_{o}^{2} Y_{\Delta} e^{\overrightarrow{\boldsymbol{n}} \theta} .
$$

From (26) it is observed that quaternion power is constant for balanced voltages and load. It is also noticeable that the expression is equivalent to the expression obtained for the complex power of this load, changing the imaginary unit $j$ for the unitary quaternion $\vec{n}$.

\section{B. Three-phase unbalanced $\Delta$ load}

Considering an RLC load submitted to a sinusoidal voltage source, Kirchhoff's voltage law implies that

$$
V_{o} \cos (\omega t)=R i(t)+L \frac{d i(t)}{d t}+\frac{1}{C} \int_{0}^{t} i(\tau) d \tau .
$$

Applying the derivative in both sides

$$
-\omega V_{o} \sin (\omega t)=R \frac{d i(t)}{d t}+L \frac{d^{2} i(t)}{d t^{2}}+\frac{1}{C} i(t) .
$$

Solving the differential equation (28), considering initial conditions that cancel the transient terms, we obtain

$$
i(t)=V_{o} Y \cos (\omega t-\theta)
$$

where

$$
\begin{gathered}
Y=\frac{1}{\sqrt{R^{2}+\left(\omega L-\frac{1}{\omega C}\right)^{2}}} \\
\theta=\tan ^{-1}\left(\frac{1}{R}\left(\omega L-\frac{1}{\omega C}\right)\right) .
\end{gathered}
$$

In fact, $Y e^{-j \theta}$ is the admittance representation of the RLC load. Note that (29) is valid for any combination of loads.

Now assuming an unbalanced $\Delta$ load with admittances $Y_{a b} e^{-j \theta_{a b}}, Y_{b c} e^{-j \theta_{b c}}$ and $Y_{c a} e^{-j \theta_{c a}}$, where $Y_{m n} e^{-j \theta_{m n}}$ is the phasor representation of the admittance between phases $m$ and $n$. Using (29), the phase currents can be written as

$$
\begin{aligned}
& i_{a b}(t)=Y_{a b} v_{a b}\left(t-\theta_{a b} / \omega\right) \\
& i_{b c}(t)=Y_{b c} v_{b c}\left(t-\theta_{b c} / \omega\right) \\
& i_{c a}(t)=Y_{c a} v_{c a}\left(t-\theta_{c a} / \omega\right)
\end{aligned}
$$

where each phase $n$ to phase $m$ voltage is given by

$$
v_{m n}(t)=\sqrt{3} v_{m}\left(t+\frac{\pi}{6 \omega}\right) .
$$

To obtain the three-phase current quaternion, we use the relations between phase currents and line currents in a $\Delta$ load, that is, $i_{a}=i_{a b}-i_{c a}, i_{b}=i_{b c}-i_{a b}$ and $i_{c}=i_{c a}-i_{b c}$. Using (16), we obtain scalar and vectorial part of the quaternion power for this case. Calculating it's scalar part

$$
\widetilde{\boldsymbol{S}}=p_{a b c}(t)=v_{a} i_{a}+v_{b} i_{b}+v_{c} i_{c} .
$$

Writing (36) in terms of phase currents and phase to phase voltages, we get

$$
\widetilde{\boldsymbol{S}}=v_{a b} i_{a b}+v_{b c} i_{b c}+v_{c a} i_{c a} .
$$

Using (32)-(34) in (37) results in

$$
\begin{array}{r}
\widetilde{\boldsymbol{S}}=Y_{a b} v_{a b}(t) v_{a b}\left(t-\theta_{a b} / \omega\right)+Y_{b c} v_{b c}(t) v_{b c}\left(t-\theta_{b c} / \omega\right) \\
+Y_{c a} v_{c a}(t) v_{c a}\left(t-\theta_{c a} / \omega\right) .
\end{array}
$$

The scalar part of $\boldsymbol{S}$ is then obtained using (12)-(14), (35) and the trigonometric identity $\cos (\alpha) \cos (\alpha-\beta)=$ $1 / 2(\cos (2 \alpha-\beta)+\cos (\beta)))$

$$
\begin{array}{r}
\widetilde{\boldsymbol{S}}=\frac{3}{2} V_{o}^{2}\left[Y_{a b} \cos \left(\theta_{a b}\right)+Y_{b c} \cos \left(\theta_{b c}\right)+Y_{c a} \cos \left(\theta_{c a}\right)\right. \\
+Y_{a b} \cos \left(2 \omega t+\frac{\pi}{3}-\theta_{a b}\right)+Y_{b c} \cos \left(2 \omega t+\pi-\theta_{b c}\right) \\
\left.+Y_{c a} \cos \left(2 \omega t-\frac{\pi}{3}-\theta_{c a}\right)\right] .
\end{array}
$$


Next, we calculate the vectorial part of quaternion power $\vec{S}$ using (18)-(21). Calculating $q_{a}(t)$

$$
q_{a}(t)=v_{b} i_{c a}+v_{c} i_{a b}-\left(v_{b}+v_{c}\right) i_{b c} .
$$

Applying (32)-(34) in (40) we get

$$
\begin{aligned}
q_{a}(t)=Y_{c a} v_{b}(t) & v_{c a}\left(t-\theta_{c a} / \omega\right)+Y_{a b} v_{c}(t) v_{a b}\left(t-\theta_{a b} / \omega\right) \\
& -Y_{b c}\left(v_{b}(t)+v_{c}(t)\right) v_{b c}\left(t-\theta_{b c} / \omega\right) .
\end{aligned}
$$

Using (35), the fact that $v_{b}+v_{c}=-v_{a}$ and the trigonometric identity $\cos (p) \cos (q)=1 / 2(\cos (p+q)+\cos (p-q)), q_{a}(t)$ is given by

$$
\begin{gathered}
q_{a}(t)=\frac{\sqrt{3}}{2} V_{o}^{2}\left[Y_{a b} \cos \left(\theta_{a b}+\frac{\pi}{2}\right)+Y_{b c} \cos \left(\theta_{b c}+\frac{\pi}{2}\right)\right. \\
+Y_{c a} \cos \left(\theta_{c a}+\frac{\pi}{2}\right)+Y_{a b} \cos \left(2 \omega t+\frac{5 \pi}{6}-\theta_{a b}\right) \\
\left.+Y_{b c} \cos \left(2 \omega t-\frac{\pi}{2}-\theta_{b c}\right)+Y_{c a} \cos \left(2 \omega t+\frac{\pi}{6}-\theta_{c a}\right)\right] .
\end{gathered}
$$

Given that $\cos (\alpha)=-\sin (\alpha-\pi / 2)$, we obtain

$$
\begin{aligned}
& q_{a}(t)=-\frac{\sqrt{3}}{2} V_{o}^{2}\left[Y_{a b} \sin \left(\theta_{a b}\right)+Y_{b c} \sin \left(\theta_{b c}\right)\right. \\
&+Y_{c a} \sin \left(\theta_{c a}\right)+Y_{a b} \sin \left(2 \omega t+\frac{\pi}{3}-\theta_{a b}\right) \\
&\left.+Y_{b c} \sin \left(2 \omega t+\pi-\theta_{b c}\right)+Y_{c a} \sin \left(2 \omega t-\frac{\pi}{3}-\theta_{c a}\right)\right] .
\end{aligned}
$$

Following an analogous procedure we conclude that $q_{a}(t)=$ $q_{b}(t)=q_{c}(t)$. So the quaternion power $\boldsymbol{S}$ can be written as

$$
\boldsymbol{S}=\widetilde{\boldsymbol{S}}-\overrightarrow{\boldsymbol{n}} \sqrt{3} q_{a}(t) .
$$

Comparing (39), (43) and (44), we can rewrite $\boldsymbol{S}$ as a sum of 6 quaternions in polar form as

$$
\begin{aligned}
\boldsymbol{S}=\frac{9}{2} \frac{Y_{a b}}{3} V_{o}^{2} e^{\overrightarrow{\boldsymbol{n}} \theta_{a b}}+\frac{9}{2} \frac{Y_{b c}}{3} V_{o}^{2} e^{\overrightarrow{\boldsymbol{n}} \theta_{b c}} \\
+\frac{9}{2} \frac{Y_{c a}}{3} V_{o}^{2} e^{\overrightarrow{\boldsymbol{n}} \theta_{c a}}+\frac{9}{2} \frac{Y_{a b}}{3} V_{o}^{2} e^{\overrightarrow{\boldsymbol{n}} \phi_{a b}(t)} \\
+\frac{9}{2} \frac{Y_{b c}}{3} V_{o}^{2} e^{\overrightarrow{\boldsymbol{n}} \phi_{b c}(t)}+\frac{9}{2} \frac{Y_{c a}}{3} V_{o}^{2} e^{\overrightarrow{\boldsymbol{n}} \phi_{c a}(t)}
\end{aligned}
$$

where $\phi_{a b}(t)=2 \omega t+\frac{\pi}{3}-\theta_{a b}, \phi_{b c}(t)=2 \omega t+\pi-\theta_{b c}$, and $\phi_{c a}(t)=2 \omega t-\frac{\pi}{3}-\theta_{c a}$.

If we calculate the usual complex power for the same load, we get

$$
S=\frac{9}{2} \frac{Y_{a b}}{3} V_{o}^{2} e^{j \theta_{a b}}+\frac{9}{2} \frac{Y_{b c}}{3} V_{o}^{2} e^{j \theta_{b c}}+\frac{9}{2} \frac{Y_{c a}}{3} V_{o}^{2} e^{j \theta_{c a}} .
$$

If the quaternion power is compared to equivalent expression for the usual complex power, it can be observed that, analogously to the balanced case, the three first terms of (45) are equivalent to the complex power, substituting $j$ for $\vec{n}$. The three last terms, on the other hand, are unique to the quaternion power representation and account for instantaneous power dynamics.

Comparing (26) and (45), it is observed that the quaternion power of an unbalanced three-phase load is equivalent to the sum of the power of six different balanced three-phase loads: three balanced consisting of $1 / 3$ of the admittances between each phase and three balanced with varying in time phases.

Summing the three constant quaternions, we can rewrite (45) as

$$
\begin{aligned}
\boldsymbol{S}=\frac{9}{2} \frac{Y_{p l}}{3} V_{o}^{2} e^{\overrightarrow{\boldsymbol{n}} \theta_{p l}}+\frac{9}{2} \frac{Y_{a b}}{3} V_{o}^{2} e^{\overrightarrow{\boldsymbol{n}} \phi_{a b}(t)} & \\
& +\frac{9}{2} \frac{Y_{b c}}{3} V_{o}^{2} e^{\overrightarrow{\boldsymbol{n}} \phi_{b c}(t)}+\frac{9}{2} \frac{Y_{c a}}{3} V_{o}^{2} e^{\overrightarrow{\boldsymbol{n}} \phi_{c a}(t)}
\end{aligned}
$$

where

$$
\begin{aligned}
& Y_{p l}^{2}=Y_{a b}^{2}+Y_{b c}^{2}+Y_{c a}^{2}+2 Y_{a b} Y_{b c} \cos \left(\theta_{a b}-\theta_{b c}\right) \\
& +2 Y_{b c} Y_{c a} \cos \left(\theta_{b c}-\theta_{c a}\right)+2 Y_{c a} Y_{a b} \cos \left(\theta_{c a}-\theta_{a b}\right)
\end{aligned}
$$

$$
\begin{aligned}
& \theta_{p l}=k_{p l} \pi \\
& +t g^{-1}\left(\frac{Y_{a b} \sin \left(\theta_{a b}\right)+Y_{b c} \sin \left(\theta_{b c}\right)+Y_{c a} \sin \left(\theta_{c a}\right)}{Y_{a b} \cos \left(\theta_{a b}\right)+Y_{b c} \cos \left(\theta_{b c}\right)+Y_{c a} \cos \left(\theta_{c a}\right)}\right)
\end{aligned}
$$

in which $k_{p l}=1$ if the denominator is negative and $k_{p l}=0$ otherwise.

These values of $Y_{p l}$ and $\theta_{p l}$ correspond to the association of the admittances in parallel, that is,

$$
Y_{p l} e^{-j \theta_{p l}}=Y_{a b} e^{-j \theta_{a b}}+Y_{b c} e^{-j \theta_{b c}}+Y_{c a} e^{-j \theta_{c a}} .
$$

The other terms can be simplified as well, which is equivalent two associate the three balanced loads with varying in time phases in parallel. The quaternion power can then be written as

$$
\boldsymbol{S}=\frac{9}{2} \frac{Y_{p l}}{3} V_{o}^{2} e^{\overrightarrow{\boldsymbol{n}} \theta_{p l}}+\frac{9}{2} \frac{Y_{\omega}}{3} V_{o}^{2} e^{\overrightarrow{\boldsymbol{n}} \phi_{\omega}(t)}
$$

where

$$
\begin{aligned}
& \phi_{\omega}=k_{\omega} \pi \\
& +t g^{-1}\left(\frac{Y_{a b} \sin \left(\phi_{a b}\right)+Y_{b c} \sin \left(\phi_{b c}\right)+Y_{c a} \sin \left(\phi_{c a}\right)}{Y_{a b} \cos \left(\phi_{a b}\right)+Y_{b c} \cos \left(\phi_{b c}\right)+Y_{c a} \cos \left(\phi_{c a}\right)}\right)
\end{aligned}
$$

in which $k_{\omega}=1$ if the denominator is negative and $k_{\omega}=0$ otherwise.

$$
\begin{aligned}
Y_{\omega}^{2}=Y_{a b}^{2}+ & Y_{b c}^{2}+Y_{c a}^{2}+2 Y_{a b} Y_{b c} \cos \left(\theta_{a b}-\theta_{b c}+\frac{2 \pi}{3}\right) \\
& +2 Y_{b c} Y_{c a} \cos \left(\theta_{b c}-\theta_{c a}+\frac{2 \pi}{3}\right) \\
& +2 Y_{c a} Y_{a b} \cos \left(\theta_{c a}-\theta_{a b}+\frac{2 \pi}{3}\right) .
\end{aligned}
$$


Notice that, if the three-phase load is balanced, $Y_{\omega}=0$.

The result in (47) can also be interpreted as the quaternionic power of one balanced three-phase impedance and three unbalanced loads with admittances between phases A-B, B-C, and $\mathrm{C}-\mathrm{A}$, respectively

$$
\frac{Y_{a b}}{3} e^{-j \theta_{a b}}, \frac{Y_{a b}}{3} e^{-j\left(2 \pi / 3+\theta_{a b}\right)} \text { and } \frac{Y_{a b}}{3} e^{j\left(2 \pi / 3-\theta_{a b}\right)}
$$

for the first three-phase impedance,

$$
\frac{Y_{b c}}{3} e^{j\left(2 \pi / 3-\theta_{b c}\right)}, \frac{Y_{b c}}{3} e^{-j \theta_{b c}} \text { and } \frac{Y_{b c}}{3} e^{-j\left(2 \pi / 3+\theta_{b c}\right)}
$$

for the second and

$$
\frac{Y_{c a}}{3} e^{-j\left(2 \pi / 3+\theta_{c a}\right)}, \frac{Y_{c a}}{3} e^{j\left(2 \pi / 3-\theta_{c a}\right)} \text { and } \frac{Y_{c a}}{3} e^{-j \theta_{c a}}
$$

for the third. This can easily be proven calculating quaternion power for each of these loads. Fig. 1 shows these loads.

Associating these admittances in parallel, we get an threephase load with followings admittances

$$
\begin{aligned}
& Y_{a b}^{\prime} e^{-j \theta_{a b}^{\prime}}= \frac{Y_{a b}}{3} e^{-j \theta_{a b}} \\
&+\frac{Y_{b c}}{3} e^{j\left(2 \pi / 3-\theta_{b c}\right)}+\frac{Y_{c a}}{3} e^{-j\left(2 \pi / 3+\theta_{c a}\right)} \\
& Y_{b c}^{\prime} e^{-j \theta_{b c}^{\prime}}= \frac{Y_{a b}}{3} e^{-j\left(2 \pi / 3+\theta_{a b}\right)} \\
&+\frac{Y_{b c}}{3} e^{-j \theta_{b c}}+\frac{Y_{c a}}{3} e^{j\left(\frac{2 \pi}{3}-\theta_{c a}\right)} \\
& Y_{c a}^{\prime} e^{-j \theta_{c a}^{\prime}=} \frac{Y_{a b}}{3} e^{j\left(2 \pi / 3-\theta_{a b}\right)} \\
&+\frac{Y_{b c}}{3} e^{-j\left(2 \pi / 3+\theta_{b c}\right)}+\frac{Y_{c a}}{3} e^{-j \theta_{c a}} .
\end{aligned}
$$

It is noticeable that (53)-(55) are not the unique possible representation of the unbalanced term of the three-phase impedance. Another representation would be obtained if we subtracted the balanced admittance from the original unbalanced three-phase admittance. For obtaining all equivalent representations of the unbalanced term, lets consider that the generic unbalanced load with admittances between phases A-B, B-C, and C-A, respectively, $M e^{-j \theta_{M}}, N e^{-j \theta_{N}}$ and $O e^{-j \theta_{O}}$. The line current of this load in parallel with the balanced load should have line currents equal to line currents of the initial unbalanced load, which implies

$$
\begin{aligned}
M e^{-j \theta_{M}}-O e^{j\left(\frac{2 \pi}{3}-\theta_{O}\right)}+ & \frac{\sqrt{3}}{3} Y_{p l} e^{-j\left(\frac{\pi}{6}+\theta_{p l}\right)}= \\
& Y_{a b} e^{-j \theta_{a b}}-Y_{c a} e^{j\left(\frac{2 \pi}{3}-\theta_{c a}\right)}
\end{aligned}
$$

$$
\begin{array}{r}
N e^{-j\left(\frac{2 \pi}{3}+\theta_{N}\right)}-M e^{-j \theta_{M}}+\frac{\sqrt{3}}{3} Y_{p l} e^{-j\left(\frac{5 \pi}{6}+\theta_{p l}\right)}= \\
Y_{b c} e^{-j\left(\frac{2 \pi}{3}+\theta_{b c}\right)}-Y_{a b} e^{-j \theta_{a b}} \\
O e^{j\left(\frac{2 \pi}{3}-\theta_{O}\right)}-N e^{-j\left(\frac{2 \pi}{3}+\theta_{N}\right)}+\frac{\sqrt{3}}{3} Y_{p l} e^{j\left(\frac{\pi}{2}-\theta_{p l}\right)}= \\
Y_{c a} e^{j\left(\frac{2 \pi}{3}-\theta_{c a}\right)}-Y_{b c} e^{-j\left(\frac{2 \pi}{3}+\theta_{b c}\right)} .
\end{array}
$$

Since (58) is a linear combination of (56) and (57), we can eliminate this equation. Solving in terms of $M e^{-j \theta_{M}}$, we obtain

$$
\begin{aligned}
N e^{-j \theta_{N}}= & M e^{j\left(\frac{2 \pi}{3}-\theta_{M}\right)}-\frac{\sqrt{3}}{3} Y_{a b} e^{j\left(\frac{\pi}{2}-\theta_{a b}\right)} \\
& +\frac{\sqrt{3}}{3} Y_{b c} e^{j\left(\frac{\pi}{6}-\theta_{b c}\right)}-\frac{\sqrt{3}}{3} Y_{c a} e^{-j\left(\frac{\pi}{6}+\theta_{c a}\right)} \\
O e^{-j \theta_{O}}= & M e^{-j\left(\frac{2 \pi}{3}+\theta_{M}\right)}-\frac{\sqrt{3}}{3} Y_{a b} e^{-j\left(\frac{\pi}{2}+\theta_{a b}\right)} \\
& +\frac{\sqrt{3}}{3} Y_{b c} e^{-j\left(\frac{5 \pi}{6}+\theta_{b c}\right)}+\frac{\sqrt{3}}{3} Y_{c a} e^{j\left(\frac{\pi}{6}-\theta_{c a}\right)} .
\end{aligned}
$$

The admittance $M e^{-j \theta_{M}}$ can be chosen arbitrarily, which implies that there are infinite equivalent unbalanced loads to the admittances shown in (53)-(55). This result was also obtained by [12], who showed that for a three wire system with balanced voltages, there are infinite equivalents to an unbalanced load. Nevertheless, the unbalanced three-phase load represented by (59) and (60) has also the propriety of null average three-phase power. This is a consequence of the expression of quaternion power (50), where the first term accounts for the average three-phase power of the load.

Returning to (50), we can simplify the expression summing both quaternions. This sum results in the shortest version of quaternion power, analogous to the balanced case presented in (26)

$$
\boldsymbol{S}=\frac{9}{2} \frac{Y_{\delta}(t)}{3} V_{o}^{2} e^{\overrightarrow{\boldsymbol{n}} \phi_{\delta}(t)}
$$

where

$$
\begin{gathered}
Y_{\delta}(t)^{2}=Y_{p l}^{2}+Y_{\omega}^{2}+2 Y_{p l} Y_{\omega} \cos \left(\theta_{p l}-\phi_{\omega}(t)\right) \\
\phi_{\delta}(t)=k_{\delta} \pi+t g^{-1}\left(\frac{Y_{p l} \sin \left(\theta_{p l}\right)+Y_{\omega} \sin \left(\phi_{\omega}(t)\right)}{Y_{p l} \cos \left(\theta_{p l}\right)+Y_{\omega} \cos \left(\phi_{\omega}(t)\right)}\right)
\end{gathered}
$$

in which $k_{\delta}=1$ if the denominator is negative and $k_{\delta}=0$ otherwise.

Analyzing (61), it is possible to conclude that the three-phase unbalanced impedance can be represented by a three-phase balanced load, with modulus and phase varying in time. 


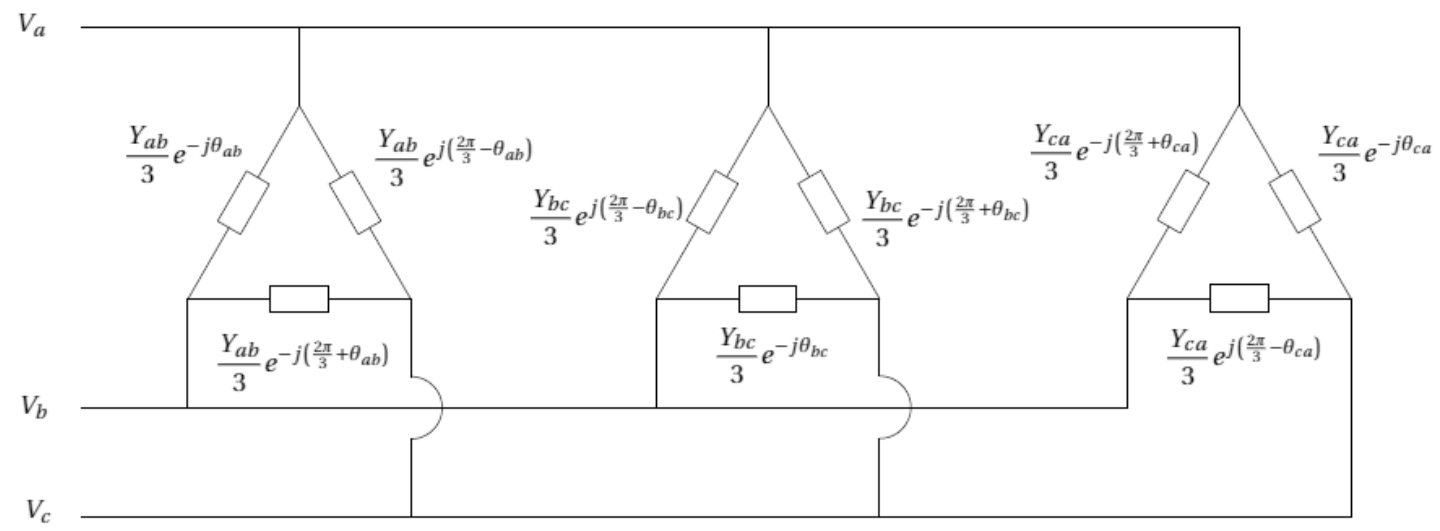

Fig. 1. Equivalent admittances

\section{Conclusions}

In this paper, power of three-phase balanced and unbalanced systems was analyzed in a quaternion framework.

The quaternion power is a characterization of the instantaneous power. It can be written in some equivalent expressions that bring useful information about the usual power and load unbalance.

We have shown that the quaternion power can be expressed with two terms: one that is equivalent to the usual complex power and other that accounts for the time-variant contribution of the load imbalance on the power. The second component of the power can be used as a measure of the load unbalance level. It is an unbiased measure in the sense that for a balanced load this second term is zero. This power decomposition also makes it natural to introduce the idea of decomposition of the unbalanced load in terms of a balanced component and an unbalanced load with null average power.

The quaternion power can be expressed as well in a form which makes clear that any unbalanced load can be represented as a time-varying balanced load.

\section{References}

[1] H. Akagi, Y. Kanazawa, and A. Nabae, "Instantaneous reactive power compensators comprising switching devices without energy storage components," IEEE Transactions on industry applications, no. 3, pp. 625630, 1984.

[2] J. L. Willems, "A New Interpretation of the Akagi-Nabae Power Components for Nonsinusoidal Three-Phase Situations," IEEE Transactions on Instrumentation and Measurement, vol. 41, no. 4, pp. 523-527, 1992.

[3] F. Z. Peng and J. S. Lai, "Generalized instantaneous reactive power theory for three-phase power systems," IEEE Transactions on Instrumentation and Measurement, vol. 45, no. 1, pp. 293-297, 1996.

[4] A. Menti, T. Zacharias, and J. Milias-Argitis, "Geometric algebra: A powerful tool for representing power under nonsinusoidal conditions," IEEE Transactions on Circuits and Systems I: Regular Papers, vol. 54, no. 3, pp. 601-609, 2007.

[5] F. G. Montoya, R. Baños, A. Alcayde, and F. M. Arrabal-Campos, "A new approach to single-phase systems under sinusoidal and non- sinusoidal supply using geometric algebra," Electric Power Systems Research, vol. 189, no. July, p. 106605, 2020.

[6] X. Dai, G. Liu, and R. Gretsch, "Generalized theory of instantaneous reactive quantity for multiphase power system," IEEE Transactions on Power Delivery, vol. 19, no. 3, pp. 965-972, 2004.

[7] A. J. Ustariz-Farfan, E. A. Cano-Plata, and S. Arias-Guzman, "Identification of protection coordination break points: A power quality approach," IEEE Industry Applications Magazine, vol. 25, no. 5, pp. 68-82, 2019.

[8] O. V. Nos, "Linear transformations in mathematical models of an induction motor by quaternions," International Workshop and Tutorials on Electron Devices and Materials, EDM - Proceedings, vol. 1, pp. 295298, 2012.

[9] N. Barry, "Electrical circuit analysis using four dimensional complex numbers, in the form of quaternions," Proceedings of the Universities Power Engineering Conference, 2013.

[10] V. D. P. Brasil, A. De Leles Ferreira Filho, and J. Y. Ishihara, "Electrical three phase circuit analysis using quaternions," Proceedings of International Conference on Harmonics and Quality of Power, ICHQP, vol. 2018-May, no. 1, pp. 1-6, 2018.

[11] O. V. Nos, A. V. Korovin, N. I. Nos, and E. S. Kucher, "Quaternion control of four-leg inverter for distribution system with harmonicproducing load," in 2020 XI International Conference on Electrical Power Drive Systems (ICEPDS), pp. 1-6, IEEE, 2020.

[12] L. Czarnecki, "Equivalent circuits of unbalanced loads supplied with symmetrical and asymmetrical voltage and their identification," Electrical Engineering, vol. 78, no. 3, pp. 165-168, 1995. 